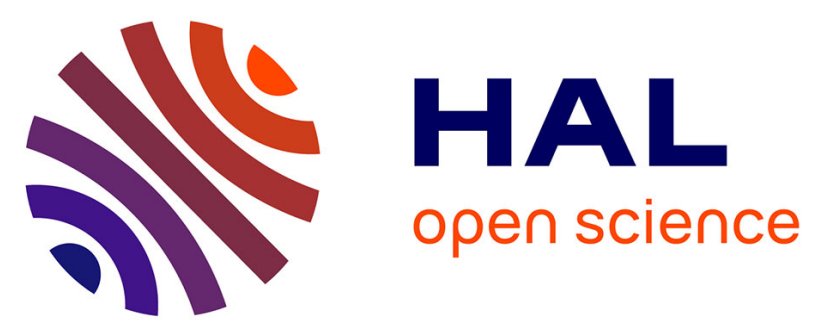

\title{
Investigation of the electron emission properties of Silver: from exposed to ambient atmosphere Ag surface to ion-cleaned Ag surface
}

T Gineste, M Belhaj, G. Teyssedre, J Puech

\section{- To cite this version:}

T Gineste, M Belhaj, G. Teyssedre, J Puech. Investigation of the electron emission properties of Silver: from exposed to ambient atmosphere Ag surface to ion-cleaned Ag surface. 13th Int. Symp. on Materials in the Space Environment (ISMSE), Pau, France, 22-26 June 2015, Jun 2015, Pau, France. pp. 1-6. hal-03033872

\section{HAL Id: hal-03033872 \\ https://hal.science/hal-03033872}

Submitted on 1 Dec 2020

HAL is a multi-disciplinary open access archive for the deposit and dissemination of scientific research documents, whether they are published or not. The documents may come from teaching and research institutions in France or abroad, or from public or private research centers.
L'archive ouverte pluridisciplinaire HAL, est destinée au dépôt et à la diffusion de documents scientifiques de niveau recherche, publiés ou non, émanant des établissements d'enseignement et de recherche français ou étrangers, des laboratoires publics ou privés. 


\title{
Investigation of the electron emission properties of Silver: from exposed to ambient atmosphere Ag surface to ion-cleaned Ag surface.
}

\author{
T Gineste ${ }^{1}$, M Belhaj ${ }^{1}$, G Teyssedre ${ }^{2}$, J Puech $^{3}$ \\ ${ }^{1}$ ONERA The French Aerospace Lab, 31055 Toulouse, France \\ ${ }^{2}$ LAPLACE - Laboratoire Plasma et Conversion d'Energie -UMR 5213 \\ Université Paul Sabatier - 118, route de Narbonne \\ 31062 Toulouse Cedex, France \\ ${ }^{3}$ CNES, 18 Avenue Edouard Belin 31055 Toulouse Cédex 9, France
}

\begin{abstract}
Electron emission properties of materials are highly dependent to the surface and the first nanometres subsurface. Technical materials, i.e used within applications are ordinarily exposed to atmosphere, which interacts with the surface. The contamination layer building up at the surface of materials and/or oxidation layer affects dramatically the electron emission proprieties. In this paper, starting from 99.99\% pure silver sample, exposed 4 years to ambient atmosphere, we monitored the variations of the electron emission properties and the surface composition during step by step ion etching procedure.
\end{abstract}

\section{INTRODUCTION / SECTION TITLE}

When a solid is hit by incident electrons, the energy transfer can result in the emission of secondary electrons (SE) and backscattered electrons (BSE). Electron emission (EE) is a physical phenomenon involved in several scientific and technical fields such as micro-analysis [1], particle accelerator [2][3] space technology (spacecraft charging [4], hall effect thrusters [5]), nuclear Physics [6]. Electron emission is also at the origin of multipactor effect that can occur in several radiofrequency (rf) devices operating under vacuum (waveguides, circulators). Multipactor effect can be defined as the generation of an electron cloud by resonance between electron emission and rf field [7]. The consequences of this adverse effect are a power limitations and additional constraints in the design of $\mathrm{rf}$ components. The estimate of its onset threshold is highly needed. In many situations, the threshold could be estimated from simulations [8]. In the framework of a multi-laboratory project[9], it was clearly established that all tested multipactor models and simulation software are extremely sensitive to the electron emission properties of the material components. Therefore, to improve the modelling of this effect and prevent occurrence, it is necessary to determine accurately some properties of the emitted electrons such as (i) the secondary electron emission yield (SEY), (ii) the electron backscattered yield (BSEY) and (iii) the energy distribution $\mathrm{N}(\mathrm{E})$. These properties are ordinarily extracted from measurements performed on materials of high purity evaporated [10] or ion cleaned [11] under ultra-high vacuum conditions (UHV). However, the materials used in rf devices are usually exposed days and months to ambient atmosphere. Thus, their surface properties are extremely different from that of the bulk. Since the emitted secondary electrons have a low energy (a few eV) and are generated in the first few nanometers in depth; obviously, the tabulated EE properties of pure materials are far from being representative of technical materials. For instance, the maximum of the electron emission yield (EEY) of pure aluminium is lower than 1 [10], while that of technical aluminium is higher than 3 [12]. The aim of this work is to study experimentally the electron emission of exposed to atmosphere silver and to establish the relation between the EE properties and the surface composition. Technical Ag surface (exposed to atmosphere) is supposed to be composed by $\mathrm{Ag}_{2} \mathrm{~S}$ [13] and other deposited compounds, like water and hydrocarbons [14]. A special experimental protocol was developed to extract relevant quantities. The electron emission yield (EEY) is investigated from very low incidence energy (some eV) to $2000 \mathrm{eV}$. A step by step Argon ion etching was performed in situ. The surface composition is monitored by Auger electron spectroscopy (AES) during cleaning process. The EEY was measured at each step. An overall drop of the EEY was observed during the ion cleaning process. The maximum of the EEY decreases from 2.26 to 1.67 and the first crossover energy increases from $18 \mathrm{eV}$ to 130 $\mathrm{eV}$ during the ion cleaning process. The effect of the ion-cleaning on the EEY tends to be suppressed after venting of the vacuum chamber. The EEY variations were linked to the surface composition.

\section{EXPERIMENTAL}

\subsection{The experimental setup}

The principle of the experimental protocol is represented in figure 1. EEY measurements, AES and electron emission spectra were recorded before and after each erosion step. Similar protocol has been used by Contini et al [15] to study electron emission loss spectra of aluminium at only three etching steps All measurements were performed in the CELESTE facility 
at ONERA. This facility is entirely dedicated and designed to the study of electron emission. A dry turbomolecular pump associated with an oil-free primary pump allows the system to be maintained at a vacuum level down to $5 \times 10^{-9}$ mbar. The tank is grounded. The sample holder allows the variation of the electron incidence angle from $0^{\circ}$ (normal incidence angle) to $60^{\circ}$. An ELG-2 electron gun from Kimball Instrument was used. The electron beam was pulsed during EEY measurements to limit the surface conditioning effect [12] and was continuous during spectra acquisition. The Tectra ion gun used has an energy range from $50 \mathrm{eV}$ to $5000 \mathrm{eV}$. Argon gas is injected though a microleak valve and atoms are excited by microwaves generated by a microwave magnetron with a $2.45 \mathrm{GHz}$ frequency. The Omicron hemispherical electron analyzer can record spectra from 2 to $2000 \mathrm{eV}$ with an accuracy of $0.5 \mathrm{meV}$. The sample was negatively biased to $-9 \mathrm{~V}$ during EEY and AES measurements to avoid experimental noise in the very first $\mathrm{eV}$ of the emission spectrum in the AES case and to avoid low energy secondary electrons to be recollected by the sample. It should be noticed that due to the $-9 \mathrm{~V}$ sample biasing, all the AES spectra are shifted by $9 \mathrm{eV}$ to high energies. Prior to measurements, the tank was baked to $180^{\circ} \mathrm{C}$ for 16 hours.

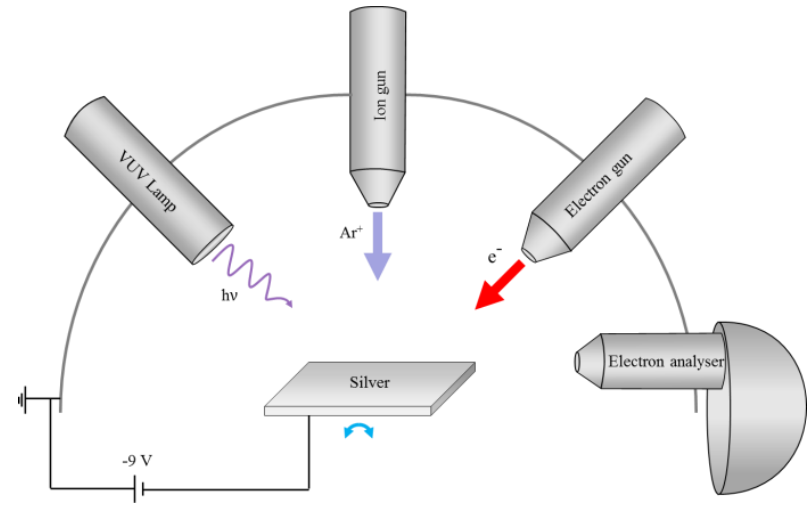

Fig. 1. Schematic representation of experimental setup CELESTE

\subsection{Sample}

The sample was $\mathrm{Ag}(\mathrm{Ag} 00470 / 31)$ of high purity (99.99\%) provided by Goodfellow Company. It was exposed for more than 4 years to ambient atmosphere. It has a square shape of $32 \times 20 \mathrm{~mm}^{2}$ area and a $0.5 \mathrm{~mm}$ thickness. Roughness measurements performed on the sample using a Zygo interferometer prior to the experiments revealed an average roughness of $160 \mathrm{~nm}$. The sample has been washed by ethanol before mounting in the measurement system, where it was outgassed in UHV for 10 days before the first measurement. It was also heated to $100^{\circ} \mathrm{C}$ for 4 hours during the UHV exposure.

\subsection{Erosion parameter}

The sample was etched with Argon ions in 14 steps.
Short ion irradiations durations were chosen for the first steps to avoid a too fast composition evolution. The irradiation duration of each step was progressively increased, starting with 30s for the first one until 30min for the last step. The same parameters (Ar ions energy of $1 \mathrm{keV}, 3,5 \mu \mathrm{Acm}^{-2}$, normal incidence) for each erosion step were applied; only the erosion duration of each step was varied. The ion current, measured using a Faraday cup was adjusted to $3.5 \mu \mathrm{A} / \mathrm{cm}^{2}$. The results presented in this paper are focused on (a) the as received sample (before erosion), (b) the sample at an intermediate state corresponding to an erosion time of $62.5 \mathrm{~min}$ and (c) the sample considered as completely cleaned after $132 \mathrm{~min}$ of erosion (final state). The final state is considered reached when both the AES spectrum, due to contaminants and EEY have reached a steady state.The thickness of the removed layer was estimated considering the etching rate (atom/ion) found in literature[16][17], Two majors hypothesis were used to estimate removed thickness as a function of the etching time:

1. The measured ion current is not totally representative of the incident Ar flux, neutral Ar may also impact the sample surface and therefore lead to underestimate of removed thickness.

2. The etching rate considered is that of pure silver. This hypothesis is partially incorrect, especially for the first etching steps when the major portion of ejected atoms are contaminants and not silver. This may lead to uncertainty in the removed thickness estimation because the etching rate of contamination layer is unknown.

\section{RESULTS}

\subsection{Surface composition}

The AES microanalysis technique was used to monitor the surface and near surface chemical composition (few nanometers in depth). After each erosion step, several AES peaks (as Ag, C, O, S) were recorded under $2 \mathrm{keV}$ electron excitation with an incident angle of $57^{\circ}$ with respect to normal incidence. No Auger peak characteristic of sulphur was observed during the entire protocol which is in contradiction with previous reports[13]. The evolution of $\mathrm{Ag}$ peak (transition MNN), C peak (transition KLL) and O peak (transition KLL) at the three main steps are represented in figures 2, 3 and 4. Each curve has been vertically shifted for sake of clarity. The evolution of the surface composition could be described according to the following four main evolutions.

$i$. On the 'as received' sample, the silver peak is weak as represented in figure 2 . This feature implies that the mean thickness of the contamination layer is comparable to the mean Auger electrons escape depth (few $\mathrm{nm}$ ). This is supported by the large carbon contamination peak 
detected on the silver surface around $265 \mathrm{eV}$. A weak oxygen peak is also observed.

ii. After the first three etching step corresponding to $1.5 \mathrm{~min}$ of erosion, oxygen is totally eliminated. This oxygen was presumably contained in thin deposited water layer on the sample as it was already reported by Baglin on several metals [12]. The $\mathrm{C}$ and $\mathrm{Ag}$ Auger peaks remain unchanged.

iii. At the intermediate state, the $\mathrm{C}$ peak was broadened to the higher energies range. This broadening implies that a chemical valance shift occurred during the cleaning process. Indeed, the carbon compounds are most probably fragmented by ion bombardment resulting in a statistical increase of the number of different chemical bound. The increase of the Ag peak is due to the partial removing of the contamination.

$i v$. At the final state, $\mathrm{C}$ peak was drastically decreased. A weak peak is still detected due to a possible implantation of carbon during ion etching and/or possible diffusion during cleaning process from the surface to the volume. At this stage, most of contaminants have been removed resulting in stable and high intensity Ag peak.

During all the cleaning process, no energy shift of the Ag peak was observed. This observation supports the fact that studied silver does not present any visible chemical bonding with any other species. Intensities of $\mathrm{Ag}, \mathrm{C}$ and $\mathrm{O}$ Auger peaks are plotted on figure 5 at every step of the protocol. Intensities are deduced from highness of Auger peak derivative. $\mathrm{C}$ and $\mathrm{O}$ are plotted following the main $\mathrm{Y}$ axis end $\mathrm{Ag}$ peak following the secondary $\mathrm{Y}$ axis.

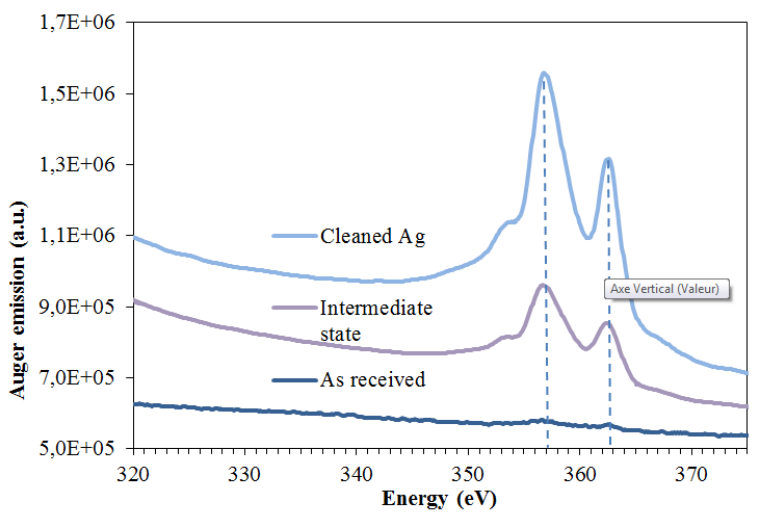

Fig. 2. Auger peak of Ag (biased-9V). As received, Intermediate and Cleaned $\mathrm{Ag}$ correspond to cumulated ion erosion times of $0,62.5$ and $132 \mathrm{~min}$.

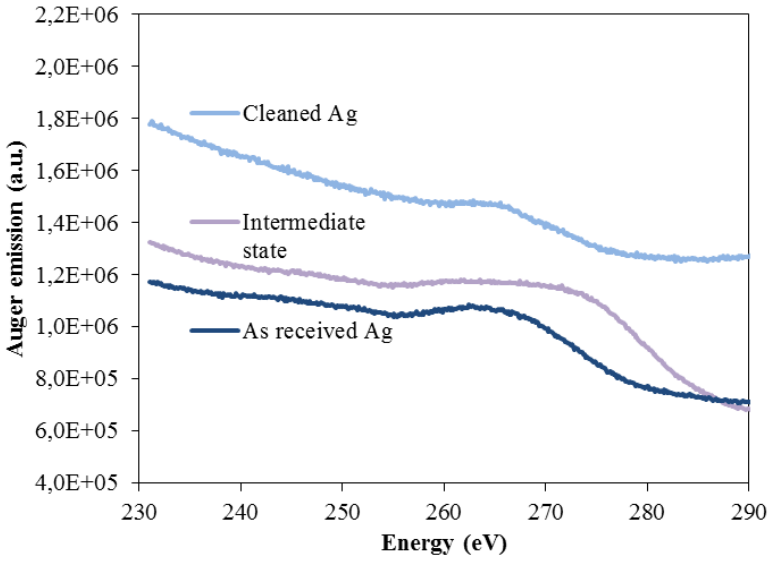

Fig. 3. Auger peak of carbon on Ag (biased -9V).. As received, Intermediate and Cleaned $\mathrm{Ag}$ correspond to cumulated ion erosion times of $0,62.5$ and $132 \mathrm{~min}$

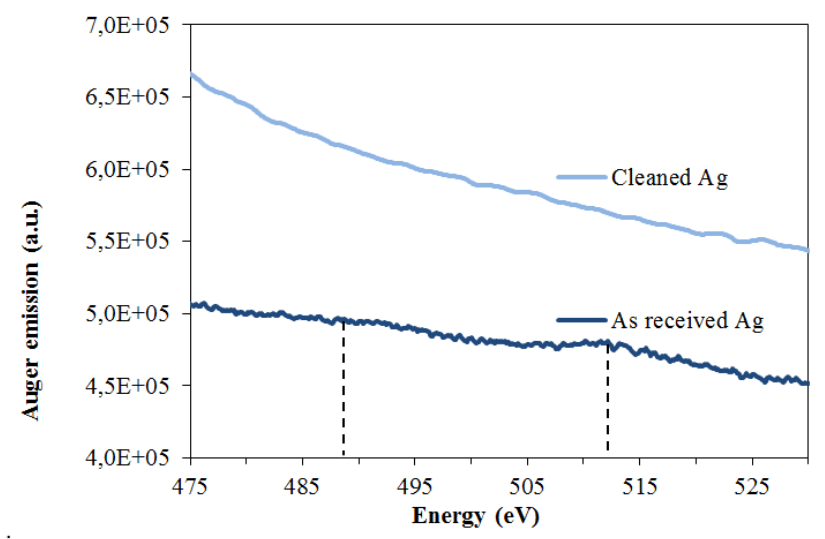

Fig. 4. Auger peak of oxygen on Ag (biased -9V). As received, Cleaned Ag correspond to cumulated ion erosion times of 0 , and $132 \mathrm{~min}$

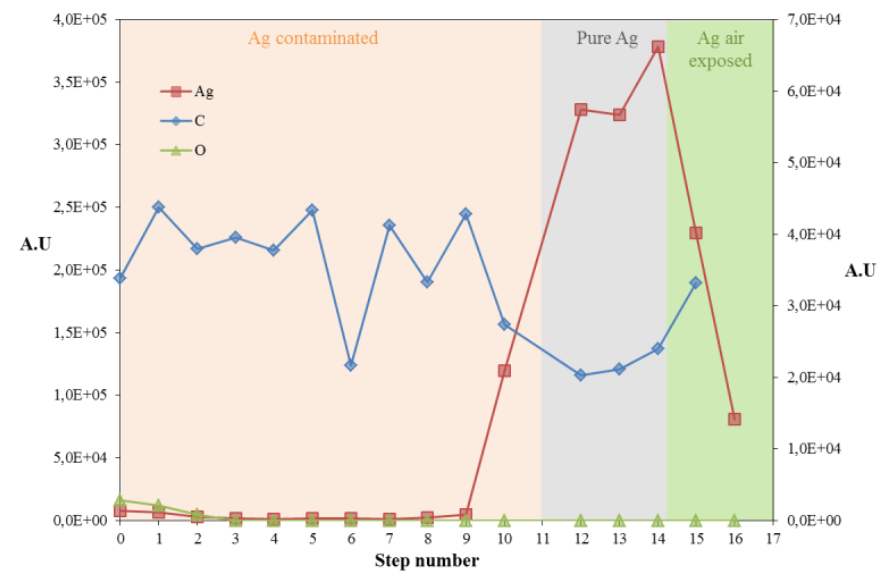

Fig. 5. Evolution of Auger peak intensity of Ag, C and $\mathrm{O}$ during the etching protocol. $\mathrm{n}^{\circ} 0$ is initial state, $\mathrm{n}^{\circ} 1$ to 14 are erosion steps and $n^{\circ} 15$ and 16 are atmosphere exposition steps. 


\subsection{Electron emission yields at initial and final state of cleaning process}

The experimental method used for EEY measurements is described in [18]. The EEY, function of the energy of primary electrons of $\mathrm{Ag}$ sample exposed to atmosphere and cleaned sample (after final erosion step) are plotted on figure $6 . \mathrm{Ec}_{1}$ at normal incidence increases from $18 \mathrm{eV}$ for as-received sample to $130 \mathrm{eV}$ for cleaned sample. The shape of the EEY curve also changes. A huge variation of the energy corresponding to the maximum of the EEY, EEY max $_{\text {ax }}$ was observed. EEYmax shifts from $300 \mathrm{eV}$ for the as received sample to $700 \mathrm{eV}$ for the cleaned one. EEY measurements of air-exposed and cleaned $\mathrm{Ag}$ are compared on figure 6 to that measured for pure $\mathrm{Ag}$ (evaporated under UHV) at normal incidence as reported by Bruining et al. [19] and by Brönstein et al. [10]. The results are in very good agreement and particularly with the curve of Brönstein et al., even if there is a divergence beyond $1500 \mathrm{eV}$. EEY values of Bruining et al. are lower but the trend is similar. These results confirm that electron emission properties of ion cleaned $\mathrm{Ag}$ sample are quite close to vacuum evaporated $\mathrm{Ag}$ and that the EEY is substantially modified by surface pollution

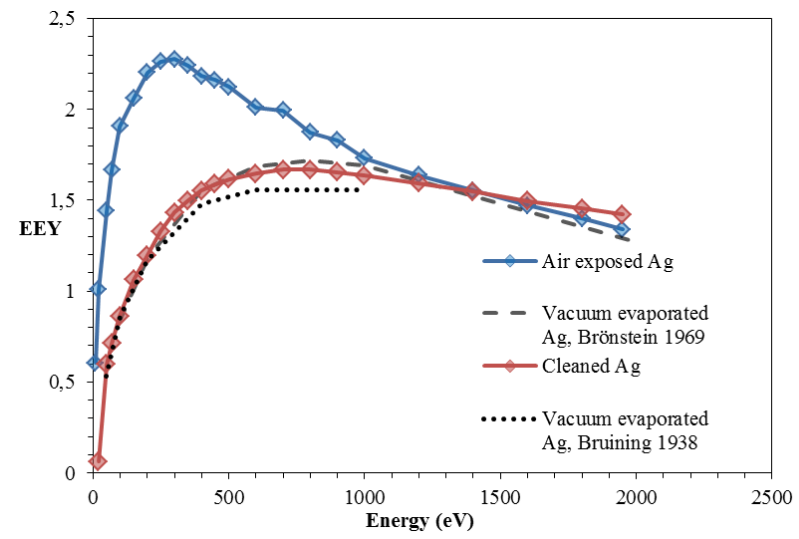

Fig. 6. Comparison of EEY of Air exposed and cleaned $\mathrm{Ag}$ to pure Ag data from the literature

\subsection{Evolution of electron emission yield during the cleaning process}

Complete EEY measurements have only been performed at the initial state and at the final state. Nevertheless, the EEY was measured at two particular incident energies during the 14 steps of the Ar etching. In order to provide an overview of the cleaning protocol effects, figure 7 shows the evolution of EEY as a function of the experiment time at incident energy of $300 \mathrm{eV}$ and after each erosion step. The estimated erosion depth is also displayed for each etching step. The eroded thickness is supposed to be directly proportional to the etching duration. i. At initial state, EEY is about 2.27 at $300 \mathrm{eV}$ incident energy. The first erosion step leads to a substantial EEY decrease of about 30\%. This critical evolution could be linked to the water layer removal that starts during this first etching step [12]. This assumption is supported by the rapid decrease of the peak O KLL.

ii. After the first erosion steps (experimental times 0 to 100h), the EEY continuously increases even if the ion sources as well as the electron gun is switched off. To explain the last results two reasons can be advocated. The first one is that the irradiation could increase the kinetic of the contamination deposition [20]. The second possible reason is that the broken and excited hydrocarbons molecules by the $\mathrm{Ar}$ bombardment are relaxed and recombined slowly between two etching steps.

iii. From the second etching step until erosion depth estimated to $21.8 \mathrm{~nm}$. The EEY measured after each step is still deceasing. For an experimental time between $240 \mathrm{~h}$ and $300 \mathrm{~h}$, the EEY is at minimum, being less than that of the cleaned $\mathrm{Ag}$ and of the contaminated Ag. An interface effect between silver and hydrocarbons contaminants seems to lower EEY and broken the linearity of the EEY evolutions from contaminated sample values to cleaned Ag EEY values. Indeed, the escape probability of low secondary electrons is highly affected by the chemical bonding of the native surface. According to the nature of the surface terminations, an upwards surface band bending may occurs resulting in a decreases in secondary electron emission through the creation of a surface barrier [21] [22]

iv. After the tenth step and an estimated removed thickness of about $42 \mathrm{~nm}$ ( $302 \mathrm{~h}$ experimental time), contamination appears as fully eliminated and the EEY is considered as characteristic of pure silver. This feature is supported by Auger electron analysis, as seen on figure 5. Once pure Ag is reached, EEY is quite stable.

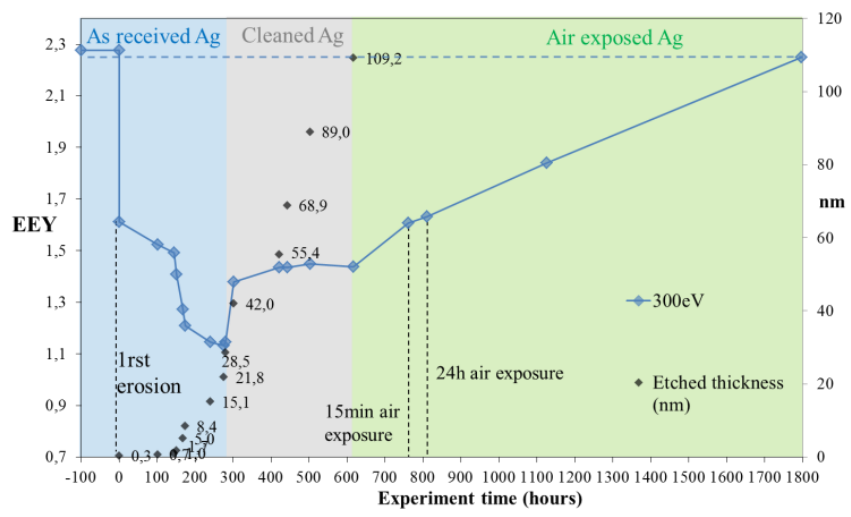

Fig. 7. Evolution of EEY at $300 \mathrm{eV}$ as a function of experiment time. Diamonds symbols represent the Arcleaning steps. Indexes correspond to the estimated erosion depth (in $\mathrm{nm}$ ) 


\subsection{Evolution of electron emission yield after exposition to ambient atmosphere.}

After the cleaning procedure of the sample, the sample was exposed to air twice. It was exposed for $15 \mathrm{~min}$, then reintroduced into the UHV chamber and exposed again to air for $24 \mathrm{~h}$ hours. Two measurements have been performed (at $760 \mathrm{~h}$ and $810 \mathrm{~h}$ ). The EEY converges to initial values due to the rapid deposition of a new contamination layer as indicate the $\mathrm{C}$ Auger peak increase after $15 \mathrm{~min}$ atmosphere exposition step $\left(\mathrm{N}^{\circ} 15\right.$ in figure 5).

\section{OUTCOMES}

We have investigated in details the step by step evolution of the EEY from as received silver sample (exposed for 4 years to ambient atmosphere) to a sample considered as pure Ag. Important evolutions of the EEY curve shape are observed during the cleaning process under Ar ions bombardment. An overall decrease of the yield was observed and in particular the first crossover energy shifts from $18 \mathrm{eV}$ (as received sample) to $130 \mathrm{eV}$ (pure sample). This huge difference could have an impact on space applications and particularly on multipacting in wave guides. When the sample is exposed to ambient atmosphere after the cleaning process, the sample recovers progressively its initial state properties in a relatively short time. This last observation may explain the fact that the EEY measured by different groups on many technical silver or silver plate samples over years are extremely stable[9]. The contamination layer built within a few days after sample processing plays the role of quite stable "passivation layer" whose surface electronic properties screen those of the silver. The presented results highlight the fact that the use of tabulated electron emission data measured or modelled very often on pure and clean materials for fundamental investigations purpose, must be used with great caution in context of applications (such as $\mathrm{rf}$ devices exposed to atmosphere).

\section{REFERENCES}

[1] H. Seiler, «Secondary electron emission in the scanning electron microscope », J. Appl. Phys., vol. 54, nº 11, p. R1-R18, nov. 1983.

[2] K. Ohmi, «Beam-Photoelectron Interactions in Positron Storage Rings », Phys. Rev. Lett., vol. 75, n $^{\circ} 8$, p. 1526-1529, août 1995.

[3] O. S. Brüning, P. Collier, P. Lebrun, S. Myers, R. Ostojic, J. Poole, et P. Proudlock, LHC Design Report. 2004.

[4] N. Balcon, D. Payan, M. Belhaj, T. Tondu, et V. Inguimbert, «Secondary Electron Emission on Space Materials: Evaluation of the Total Secondary Electron Yield From Surface Potential Measurements », IEEE Trans. Plasma Sci., vol.
40, n⿳0 2, p. 282, 2012.

[5] A. Dunaevsky, Y. Raitses, et N. J. Fisch, «Secondary electron emission from dielectric materials of a Hall thruster with segmented electrodes », Phys. Plasmas 1994-Present, vol. 10, $\mathrm{n}^{\mathrm{o}} 6$, p. 2574-2577, juin 2003.

[6] J. P. Gunn, «Evidence for strong secondary electron emission in the tokamak scrape-off layer », Plasma Phys. Control. Fusion, vol. 54, $\mathrm{n}^{\circ}$ 8, p. 085007, août 2012.

[7] J. B. Sombrin, « Le claquage hyperfréquences et l'effet multipactor dans les satellites », 1993.

[8] M. Buyanova, V. E. Semenov, D. Anderson, M. Lisak, et J. Puech, "Influence of secondary emission yield on the saturation properties of multipactor discharges between two parallel metal plates », Phys. Plasmas 1994-Present, vol. 17, n 4, p. 043504, avr. 2010.

[9] J. Puech, C. . Miquel-Espana, D. Raboso, et EVEREST consortium, «Synthesis of the results of the EVEREST project », Proceding 8th Int. Work. Multipactor Corona Passive Intermodulation Mulcopim14, 2014.

[10] I. M. Bronshtein et B. S. Fraiman, VTORICHNAYA ELEKTRONNAYA EMISSIYA. (Secondary Electron Emission). Nauka,Moskva, 1969.

[11] A. M. D. Assa'd et M. M. El Gomati, «Backscattering coefficients for low energy electrons », Scanning Microsc., vol. 12, p. 185192, 1998.

[12] V. Baglin, J. Bojko, O. Gröbner, B. Henrist, N. Hilleret, C. Scheuerlein, et M. Taborelli, «The secondary electron yield of technical materials and its variation with surface treatments », in Proc. of EPAC, 2000, p. 217.

[13] G. J. Russ, «Characteristics of Contacts Contaminated With Silver Sulfide Film », IEEE Trans. Parts Electr. Mater. Packag., vol. PMP-6, $\mathrm{n}^{\circ} 4,1970$.

[14] M. Amodio, S. Catino, P. R. Dambruoso, G. de Gennaro, A. Di Gilio, P. Giungato, E. Laiola, A. Marzocca, A. Mazzone, A. Sardaro, et M. Tutino, «Atmospheric Deposition: Sampling Procedures, Analytical Methods, and Main Recent Findings from the Scientific Literature », Adv. Meteorol., vol. 2014, p. e161730, juin 2014.

[15] V. Contini, C. Presilla, F. Sacchetti, et S. Tosto, «Auger electron spectroscopy study of the interface between bulk aluminum and bulk aluminum oxide », Surf. Sci., vol. 276, n ${ }^{\circ} 1-3$, p. 50-58, oct. 1992.

[16] R. Behrisch et W. Eckstein, Sputtering by Particle Bombardment: Experiments and Computer Calculations from Threshold to $\mathrm{MeV}$ Energies. Springer Science \& Business Media, 2007.

[17] P. D. H. Oechsner, «Untersuchungen zur 
Festkörperzerstäubung bei schiefwinkligem Ionenbeschuß polykristalliner Metalloberflächen im Energiebereich um $1 \mathrm{keV} »$, Z. Für Phys., vol. 261, no 1, p. 37-58, févr. 1973.

[18] T. Gineste, M. Belhaj, G. Teyssedre, N. Balcon, et J. Puech, «A novel experimental setup for the measurement electron backscattering yield», Meas. Sci. Technol., vol. 25, nº 8, p. 085601, août 2014.

[19] H. Bruining et J. H. De Boer, «Secondary electron emission: Part I. Secondary electron emission of metals », Physica, vol. 5, n ${ }^{\circ} 1$, p. $17-$ 30, janv. 1938.

[20] A. Al-Ajlony, A. Kanjilal, M. Catalfano, S. S. Harilal, et A. Hassanein, « Electron irradiationenhanced water and hydrocarbon adsorption in EUV lithography devices », Appl. Surf. Sci., vol. 289, p. 358-365, janv. 2014.

[21] A. Stacey, S. Prawer, S. Rubanov, R. Ahkvlediani, S. Michaelson, et A. Hoffman, «The effect of temperature on the secondary electron emission yield from single crystal and polycrystalline diamond surfaces », Appl. Phys. Lett., vol. 95, n 26, p. 262109, déc. 2009.

[22] E. Wang, I. Ben-Zvi, T. Rao, D. A. Dimitrov, X. Chang, Q. Wu, et T. Xin, «Secondary-electron emission from hydrogen-terminated diamond: Experiments and model », Phys. Rev. Spec. Top. Accel. Beams, vol. 14, $\mathrm{n}^{\circ} 11$, p. 111301, nov. 2011. 